\title{
Neuromorphic Data Microscope
}

\author{
David R. Follett \\ Lewis Rhodes Laboratories \\ Concord, MA 01742 \\ drfollett@earthlink.net \\ Gabe D. Karpman \\ Lewis Rhodes Laboratories \\ Concord, MA 01742 \\ gdkar@alum.mit.edu
}

\author{
Duncan Townsend \\ Lewis Rhodes Laboratories \\ Concord, MA 01742 \\ work@duncancmt.com \\ John H. Naegle \\ Sandia National Laboratories \\ P.O. Box 5800 \\ Albuquerque, NM 87185 \\ jhnaegl@sandia.gov
}

\author{
Pamela L. Follett \\ Lewis Rhodes Laboratories \\ Concord, MA 01742 \\ plfollett@earthlink.net
}

\author{
Roger A. Suppona \\ Sandia National Laboratories \\ P.O. Box 5800 \\ Albuquerque, NM 87185 \\ rasuppo@sandia.gov
}

\author{
James B. Aimone \\ Sandia National Laboratories \\ P.O. Box 5800 \\ Albuquerque, NM 87185 \\ jbaimon@sandia.gov
}

\author{
Conrad D. James \\ Sandia National Laboratories \\ P.O. Box 5800 \\ Albuquerque, NM 87185 \\ cdjame@sandia.gov
}

\begin{abstract}
In 2016, Lewis Rhodes Labs, (LRL), shipped the first commercially viable Neuromorphic Processing Unit, (NPU), branded as a Neuromorphic Data Microscope (NDM). This product leverages architectural mechanisms derived from the sensory cortex of the human brain to efficiently implement pattern matching. LRL and Sandia National Labs have optimized this product for streaming analytics, and demonstrated a 1,000x power per operation reduction in an FPGA format. When reduced to an ASIC, the efficiency will improve to $1,000,000 x$. Additionally, the neuromorphic nature of the device gives it powerful computational attributes that are counterintuitive to those schooled in traditional von Neumann architectures. The Neuromorphic Data Microscope is the first of a broad class of brain-inspired, time domain processors that will profoundly alter the functionality and economics of data processing. ${ }^{1}$
\end{abstract}

\section{CCS CONCEPTS}

- Security and privacy Intrusion/anomaly detection and malware mitigation • Security and privacy Network security - Theory of computation $\sim$ Streaming models • Computer systems organization Neural networks • Computer systems organization Real-time system architecture $\cdot$ Computer systems organization $\sim$ Data flow architectures

(C) 2017 Association for Computing Machinery. ACM acknowledges that this contribution was authored or co-authored by an employee, contractor or affiliate of the United States government. As such, the United States Government retains a nonexclusive, royalty-free right to publish or reproduce this article, or to allow others

\section{KEYWORDS}

Neuromorphic Computing, Field Programmable Gate Array

\section{ACM Reference format:}

David R. Follett, Duncan Townsend, Pamela L. Follett, Gabe D. Karpman, John H. Naegle, Roger A. Suppona, James B. Aimone, Conrad D. James. 2017 Neuromorphic Data Microscope. In Proceedings of Neuromorphic Computing Symposium, Knoxville, TN, USA July 17-19, 2017 (NCS '17), 6 pages. https://doi.org/10.1145/3183584.3183617

\section{INTRODUCTION}

Developing a commercially viable neural-inspired computer requires not only understanding and application of neuroscience elements, but identification of which neural attributes to disregard. Furthermore, deeply held principals of computer architecture and processing needed to be abandoned in order for applied neurological concepts to generate a compelling paradigm shift.

Decisions in computer architecture, connectivity and processing are most advantageous if brain features are selected with consideration to the specific problem-solving goals. Tools for neuroscience and health advances or streaming data analysis will

to do so, for Government purposes only. NCS '17, July 17-19, 2017, Knoxville, TN, USA

(C) 2017 Association for Computing Machinery. ACM ISBN 978-1-4503-6442-3/17/07 ...\$15.00 https://doi.org/10.1145/3183584.3183617 
likely require divergent strategies. Forcing a similar end structure could optimize for one or the other, or might compromise the potential of both. The current set of problems that are poorly addressed by modern computing contains disparate issues unlikely to indicate a single solution. The human brain handles such problems differently and one would wish to expect no less from a neural-inspired solution.

\section{BACKGROUND AND MOTIVATION}

\subsection{Related Work}

The history of brain inspired computing dates back 70 years to the early work of McCulloch \& Pitts on Artificial Neural Nets (ANNs) [1]. Over the ensuing years there have been three major bubbles of activity. The first, spanning the mid 1950s -1970 s, was focused around natural language and search algorithms. The funding collapsed largely because the core algorithms suffered from combinational explosions that made application impractical [2]. The second bubble spanned the 1980s and leveraged advances such as Hopfield Nets [3], Lisp [4], and "expert systems". This bubble consumed in excess of $\$ 2 \mathrm{~B}$ in funding worldwide with multiple corporations introducing commercial "Lisp Machines". Like the first AI wave the technology was not commercially compelling and the market collapsed. The current AI wave was triggered by Hinton and Salakhutdinov's 2006 demonstration that each layer in a deep ANN could be effectively treated as an unsupervised restricted Boltzmann machine [5]. Although deep learning leverages neuroscience insights dating to the 1950's such as Hubel and Wiesel's experiments in the visual cortex [6] the convergence with cloud computing and big data has been critical.

Most NPU designs fall into two major classes, vector matrix multiply and spiking. Vector matrix multiply underlies most deep learning applications and is maturing rapidly. These systems accelerate ANNs using dense floating point architectures, are widely deployed in research environments [7], and have enabled an impressive list of demonstrations. A primary strength is their ability to "learn" from large data sets using re-enforcement techniques but they suffer from the classic ANN issues of low state density [8-10] and high-power consumption. A number of companies have developed or are offering products including Google [11] and NVIDIA [12], yet broad commercial viability is not yet known.

Spiking NPUs use electronic "action potentials" to communicate between processing elements such as integrate and fire neurons [13]. Unlike vector-matrix multiply machines, they incorporate a number of key neurological attributes including sparse connectivity and time-encoded spikes. Spiking NPUs have the potential to achieve dramatically higher state density at a fraction of the power relative to vector matrix multiply NPUs. IBM's True North technology is the best known commercially available spiking NPU [14].

\subsection{Neuromorphic Inspiration}

As noted earlier the brain is exceedingly diverse in terms of structure, function and dynamics. The following neuronal attributes are fundamental to the Neuromorphic Data Microscope (NPM),
Time: Medical research into white matter disease and modeling of the hippocampus has indicated the fundamental role time plays in neuronal computation [15-17]. Subtle changes in white matter resulting from injuries such as cerebral palsy or concussion can profoundly alter cognition. Relative time is to cognition what relative magnitude is to computer science.

Sparse connectivity: Power consumption in modern microprocessors is dominated by signaling, not logic. Neurological systems severely bound the degree, distance and rate of signaling.

Sparse representation: Conservative estimates of the information density of the human brain indicate that there are at least several orders of magnitude more bytes then synapses. Neuronal systems use sparse representation to achieve this state density.

We excluded hardware support for learning in the NPM. From a neurology/neuroscience perspective learning is hellishly complex. Several of the authors have extensive experience modeling complex neurological learning algorithms. Learning was externalized to enhance the general applicability and reduce the complexity of the hardware.

Implementation of brain inspired structure, function and dynamics is complicated by the profound material science differences between a carbon-based brain and a silicon based processor. A short list includes, noise characteristics, signal propagation, energy transfer and thermal stability. Because of the extreme dissimilarity functionally equivalent architectures typically have profoundly different structure and dynamics.

\section{RESULTS AND DISCUSSION}

\subsection{Neuromorphic Data Microscope Architecture}

The von Neumann architecture includes a complex central processing unit coupled to a flat memory space. The resulting system is Turing complete and the fundamental building block of the electronic revolution. Like the brain, LRL's Neuromorphic Data Microscope inverts this model. The current design is implemented on an Intel Arria 101150 FPGA and contains 4,255 abstracted neurons, called Processing Elements (PEs), layered on a non-uniform memory. PEs are sparsely interconnected with a proximity bias; the vast majority of connections are sub-millimeter and point to point, i.e. low capacitance. As a result, the NPU is easily clocked at $235 \mathrm{MHz}$ and consumes <16W @ 48oC. Design work using TSMC's $16 \mathrm{~nm}$ processes indicates an ASIC implementation could support 100,000 abstracted neurons clocked at $2.5 \mathrm{GHz}$ consuming roughly $50 \mathrm{~W}$ of power. Further, freed from FPGA architectural constraints significant PE optimization becomes possible.

The current Neuromorphic Data Microscope design uses a single PE type, although PEs with unique characteristics are easily designed and introduced. Like the sensory cortex, production Neuromorphic Data Microscopes are optimized for pattern matching and are not Turing complete. Operationally, individual patterns are analyzed using multiple PEs, while individual PEs contribute to the analysis of multiple patterns. The ability to decompose problems in terms of space and time is fundamental to 
the performance characteristics of both the brain and these novel devices.

The Data Microscope has interesting attributes that derive directly from the neuroscience including:

1. Extremely low power consumption, size, and weight

2. Efficient analysis of temporal and spatial pattern variants. For instance, it is more efficient for a neuromorphic processor to evaluate a pattern against all time variants than a specific one.

3. Almost no overhead for context switching the processor state

4. Dynamic programmability, enabling efficient execution of adaptive algorithms

5. Deterministic performance that is not dependent on the patterns being evaluated or the content of the data stream

6. Behavioral analysis capabilities. NPUs can often detect "concept drift", sometimes call "evolving threats", with a bounded degree of certainty.

\subsection{Compiler}

Compatibility with existing infrastructure is critical to the commercial success of the Neuromorphic Data Microscope. This is achieved through a traditional, high performance API that incorporates a first-generation compiler for standards-compliant Perl Compatible Regular Expressions, (PCRE). Like traditional compilers, the input source is transformed into a graph representation, however, optimization and reduction is greatly complicated by the non-uniform memory structure. In essence, optimization becomes a complex, multi-variable, challenge as proper execution is dependent on both order and location.

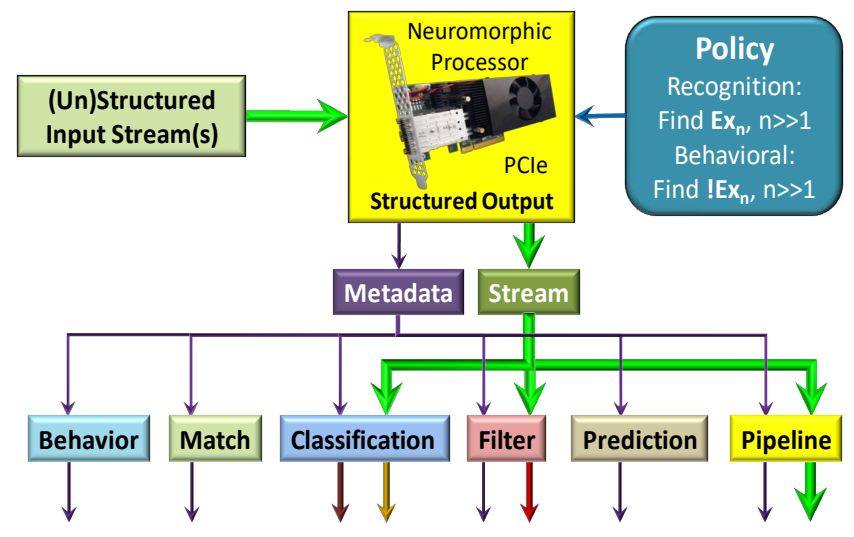

Figure 1: Summary of Neuromorphic Data Microscope system level functionality.

\subsection{Neuromorphic Data Microscope}

The Neuromorphic Data Microscope is a general purpose, PCIebased slave device for streaming data analytics. In operation, an application supplies a policy(s) that articulate a combination of behavioral or detection criteria. The policy effectively defines a structure that the NPU applies against a stream(s) of (un)structured data. The output consists of meta data that correlates the new structure to the stream. The power of the device is its ability to efficiently execute extremely complex policies, relative to current state-of-the-art solutions. Applications for the Neuromorphic Data Microscope are very broad and include: cyber security, filtering, classification, switching, and behavioral prediction, Figure 1.

\subsection{Sample Commercial Application: Cyber Defense}

At scale, cyber defense is a three-stage process, Figure 2. First, sensors monitor network and system activity for malicious events and generate alerts based on deployed security policies. Policies contain cyber event descriptions frequently in the form of Snort rules. However, Cyber events that are not easily defined by a static signature may be better described within Perl Compatible Regular Expressions (PCRE). Second, alerts are consolidated into an Operational Intelligence system (e.g. Splunk). This system provides an integrated view of potential threats and buffers the analysts from the alert generation process. Finally, highly trained security analysts mine the Operational Intelligence system, relying upon experience and a variety of analytic tools to identify and neutralize threats.

From the perspective of a cyber analyst the architecture has three fundamental weaknesses. First, the fidelity of the sensorgenerated alert data is excruciatingly low, placing a significant burden on the Operational Intelligence system and analyst community that uses it. Second, the system frequently fails to identify false positives because it's unable to evaluate common predicted behaviors, is slow in adapting to evolving threats, and rich sensor fields are expensive to deploy and support. Third, the high computational expense of PCREs compels analysts to limit their use or risk missing network events due to excessive CPU consumption; a "risk vs. reward" problem analysts must carefully manage.

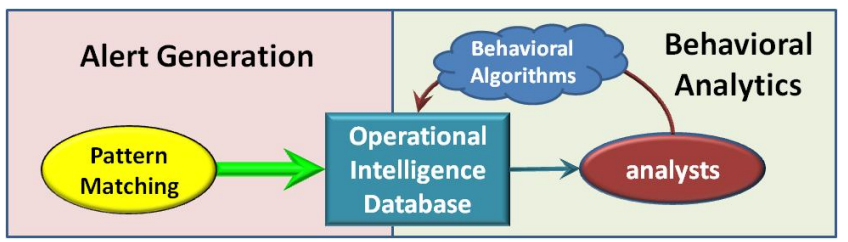

Figure 2: State-of-the-art cyber defense is a 3 stage process; 1) alert generation, 2) data consolidation and 3) analyst data mining often with behavioral algorithms.

\subsection{Benchmarking Methodology}

While it's possible to directly benchmark the Neuromorphic Data Processor against similar functional units such as RE2 (https://github.com/google/re2),

Hyperscan (https://01.org/hyperscan), or Micron's Automata processor [18] these benchmarks only loosely correlate to the system level impact. There are a number of reasons for this including,

1. The characteristics of neuromorphic computation differ significantly from legacy approaches. For instance, the NDM is most efficient when evaluating all expressions against all possible 
locations while current technology includes "directives" that save computational resources by limiting the range of evaluation.

2. The relative performance gain for individual expressions varies significantly.

3. The throughput of alternate approaches is a function of the data stream security policy while the NDM is deterministic up to its theoretical maximum.

4. NDMs can generate alerts at a rate that endangers the stability of cyber defense systems such as the one shown in figure 2 . The current design leverages the neuromorphic nature of the technology to protect against this scenario.

5. NDMs uniquely support "behavioral" algorithms that are highly leveraged at the system level.

6. NDMs offload multiple CPU intensive system functions such as sessionization.

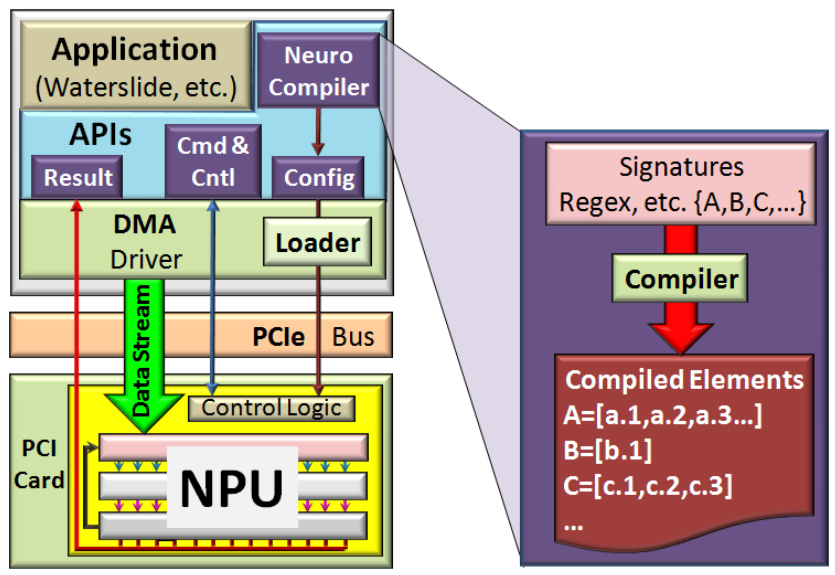

Figure 3: Neuromorphic complexity is hidden by a complex software stack.

Consequently, we measure performance at the system application level using WaterSlide, a deployed streaming analytics application (www.github.com/waterslideLTS/waterslide) which includes several standard pattern matching algorithms including RE2. The modular nature of WaterSlide enabled us to easily port the NDM using the software stack and API shown in Figure 3. Performance is evaluated by providing a security policy and data stream to WaterSlide and measuring the relative speed of execution under RE2 and the Neuromorphic Data Microscope. The timing delay is subsequently divided by the power consumed by either the CPU in the case of RE2 or the FPGA in the case of the NDM to yield the ratio. The NDM does not take into account driver overhead but at $<15 \%$ of a core this is small. The expressions used to generate the security policy are culled from the Emerging Threats database (www.emergingthreats.net). Expression accuracy is validated by automatically deriving a trigger sequence for each expression which is randomly fuzzed to generate 2,048 test strings. The acceptance criteria requires that all strings generate identical results under RE2 and the NDM. The validated expression set contains 4,250 entries as of the latest product release. The source data stream is the publicly available https://pen- testing.sans.org/holiday-challenge/2013. All tests were run on an Intel Xeon E5-1650 V3 (6 core HT, $15 \mathrm{MB}$ cache, $3.5 \mathrm{GHz}$ ) consuming roughly $350 \mathrm{~W}$.

\subsection{Results}

A randomly chosen policy containing 446 entries was chosen from the full validated expression set. Using the methodology described above the ratio of Waterslide RE2 over Waterslide NDM is $1,934 \mathrm{x}$. A second policy optimized for the maximum number of expressions that fit in the NDM resulted in a 1,212 expression policy with a performance ratio of 3,350x. Similarly, selecting the most resource intensive expressions a policy containing 156 entries resulted in a ratio of $1,215 \mathrm{x}$.

\section{CONCLUSIONS}

In spite of the impressive results the NDM is still in its infancy. Within the next few months the performance numbers will improve by $50 \%$ based on planned compiler optimizations. We are also in the early days of introducing behavioral algorithms. Finally, the technology is extremely amenable to ASIC design where architectural, density and frequency improvements will result in roughly a further $1,000 \mathrm{x}$ improvement in operations/W. Pattern matching is pervasive in markets ranging from financial, to social media, to government. The Neuromorphic Data Microscope recently introduced by LRL profoundly alters the economics of this function by leveraging concepts derived directly from the neuroscience of the brain's sensory cortex. These devices offer unparalleled power, size, weight, and cost benefits over traditional architectures.

\section{ACKNOWLEDGMENTS}

The authors acknowledge financial support from Sandia National Laboratories' Laboratory Directed Research and Development Program, and specifically the Hardware Acceleration of Adaptive Neural Algorithms (HAANA) Grand Challenge Project. Sandia National Laboratories is a multimission laboratory managed and operated by National Technology and Engineering Solutions of Sandia LLC, a wholly owned subsidiary of Honeywell International Inc., for the U.S. Department of Energy's National Nuclear Security Administration under contract DE-NA0003525.

\section{REFERENCES}

[1] W.S. McCulloch, W. Pitts, A logical calculus of the ideas immanent in nervous activity, The bulletin of mathematical biophysics 5(4) (1943) 115-133.

[2] S. Russell, P. Norvig, A. Intelligence, A modern approach, Artificial Intelligence. Prentice-Hall, Egnlewood Cliffs 25 (1995) 27.

[3] J.J. Hopfield, Neural networks and physical systems with emergent collective computational abilities, Proceedings of the national academy of sciences 79(8) (1982) 2554-2558. 
[4] D. Weinreb, D. Moon, The lisp machine manual, ACM SIGART Bulletin (78) (1981) 10.

[5] G.E. Hinton, R.R. Salakhutdinov, Reducing the dimensionality of data with neural networks, science 313(5786) (2006) 504-507.

[6] D.H. Hubel, T.N. Wiesel, Receptive fields of single neurones in the cat's striate cortex, The Journal of physiology 148(3) (1959) 574-591.

[7] S. Agarwal, S.J. Plimpton, D.R. Hughart, A.H. Hsia, I. Richter, J.A. Cox, C.D. James, M.J. Marinella, Resistive memory device requirements for a neural algorithm accelerator, Neural Networks (IJCNN), 2016 International Joint Conference on, IEEE, (2016) 929-938.

[8] C.-Y. Liou, S.-K. Yuan, Error tolerant associative memory, Biological Cybernetics 81(4) (1999), 331-342.

[9] C.-Y. Liou, S.-L. Lin, Finite memory loading in hairy neurons, Natural Computing 5(1) (2006) 15-42.

[10] E. Gardner, Maximum storage capacity in neural networks, EPL (Europhysics Letters) 4(4) (1987) 481.

[11] N.P. Jouppi, C. Young, N. Patil, D. Patterson, G. Agrawal, R. Bajwa, S. Bates, S. Bhatia, N. Boden, A. Borchers, Indatacenter performance analysis of a tensor processing unit, arXiv preprint arXiv:1704.04760 (2017).

[12] N. Bell, M. Garland, Efficient sparse matrix-vector multiplication on CUDA, Nvidia Technical Report NVR2008-004, Nvidia Corporation, (2008).

[13] M.R. Smith, A.J. Hill, K.D. Carlson, C.M. Vineyard, J. Donaldson, D.R. Follett, P.L. Follett, J.H. Naegle, C.D. James, J.B. Aimone, A novel digital neuromorphic architecture efficiently facilitating complex synaptic response functions applied to liquid state machines, Neural Networks (IJCNN), 2017 International Joint Conference on, IEEE, 2017, 2421-2428.

[14] F. Akopyan, J. Sawada, A. Cassidy, R. Alvarez-Icaza, J. Arthur, P. Merolla, N. Imam, Y. Nakamura, P. Datta, G.-J. Nam, Truenorth: Design and tool flow of a $65 \mathrm{mw} 1$ million neuron programmable neurosynaptic chip, IEEE Transactions on Computer-Aided Design of Integrated Circuits and Systems 34(10) (2015) 1537-1557.

[15] P.L. Follett, P.A. Rosenberg, J.J. Volpe, F.E. Jensen, NBQX attenuates excitotoxic injury in developing white matter, Journal of Neuroscience 20(24) (2000) 9235-9241.

[16] P.L. Follett, W. Deng, W. Dai, D.M. Talos, L.J. Massillon, P.A. Rosenberg, J.J. Volpe, F.E. Jensen, Glutamate receptor-mediated oligodendrocyte toxicity in periventricular leukomalacia: a protective role for topiramate, Journal of Neuroscience 24(18) (2004) 44124420.

[17] P.L. Follett, C. Roth, D. Follett, O. Dammann, White matter damage impairs adaptive recovery more than cortical damage in an in silico model of activity-dependent plasticity, Journal of child neurology 24(9) (2009) 12051211.

[18] P. Dlugosch, D. Brown, P. Glendenning, M. Leventhal, H. Noyes, An efficient and scalable semiconductor architecture for parallel automata processing, IEEE Transactions on Parallel and Distributed Systems 25(12) (2014) 3088-3098. 